\title{
Interpretation of the coherency matrix for three-dimensional polarization states
}

\author{
José J. Gil* \\ Facultad de Educación, Universidad de Zaragoza, Pedro Cerbuna 12, 50009 Zaragoza, Spain
}

(Received 2 August 2014; published 29 October 2014)

\begin{abstract}
From an appropriate parameterization of the three-dimensional (3D) coherency matrix $\mathbf{R}$ that characterizes the second-order, classical states of polarization, the coherency matrices are classified and interpreted in terms of incoherent decompositions. The relevant physical quantities derived from $\mathbf{R}$, such as the intensity, degree of polarimetric purity, the indices of polarimetric purity, angular momentum, degree of directionality, and degree of linear polarization, are identified and interpreted in light of the case study performed. The information provided by $\mathbf{R}$ about the direction of propagation is clarified and it is found that coherency matrices with rank $\mathbf{R}=2$ do not always represent states with a well-defined direction of propagation. Moreover, the existence of 3D mixed states that cannot be decomposed into a superposition of a pure state, a two-dimensional (2D) unpolarized state, and a 3D unpolarized state is demonstrated. Appropriate representation and interpretation for all the different types of $3 \mathrm{D}$ coherency matrices are provided through physically consistent criteria. Under the approach proposed, the conventional 2D model arises naturally.
\end{abstract}

DOI: 10.1103/PhysRevA.90.043858

PACS number(s): 42.25.Ja

\section{INTRODUCTION}

A proper description of the polarization properties of electromagnetic waves relies on the concept of the coherency matrix, which is applicable regardless of the particular band of the electromagnetic spectrum considered.

The study and characterization of three-dimensional (3D) states of polarization is a subject of high interest from both theoretical and experimental points of view. Several relevant contributions have been published, mostly in recent years, concerning aspects such as the interpretation of physical quantities derived from the eigenvalues of the coherency matrix R [1-20]; geometric interpretation of 3D states [21-27]; 3D coherency matrix for plane waves and coherent composition of pure (or totally polarized) states [28-30]; incoherent composition and decomposition of pure and mixed states [7,10,13,19,25,31,32]; 3D polarimetry [33,34]; statistical and coherence properties of 3D states [9,28,35-38]; generalized Stokes parameters [2,9,13,21,24,39,40]; polarization dynamics [41,42], etc.

However, additional effort is needed in order to get answers to questions such as how to represent 3D states of polarization as combinations of states with a simple physical interpretation. Generally, unitary transformations of the coherency matrix do not correspond to rotations of the $3 \mathrm{D}$ laboratory reference frame. Therefore, which unitary transformations are physically realizable? What kind of information about the propagation direction can be obtained from the coherency matrix? How do we interpret $3 \mathrm{D}$ polarization states in terms of meaningful physical quantities? How many different physical situations can be distinguished and how do we classify them?

The aim of this work is to provide appropriate and consistent responses to the previous questions by means of (1) an adequate parameterization of the $3 \mathrm{D}$ coherency matrix $\mathbf{R}$ in terms of nine physical parameters; (2) the case study of the different physical situations, identified through specific descriptors and analyzed by means of the arbitrary and characteristic

\footnotetext{
*ppgil@unizar.es
}

decompositions of $\mathbf{R}$; and (3) the identification, definition, and interpretation of parameters that provide complete and meaningful physical information. In the remainder of this section, we summarize some concepts that are particularly useful for the development and interpretation of the results presented in later sections.

The coherency matrix or polarization matrix, which contains all measurable second-order information about the state of polarization (including intensity) of an electromagnetic wave at a given point $\mathbf{r}$, is defined as the $3 \times 3$ Hermitian matrix $\mathbf{R}=\left\langle\varepsilon(t) \otimes \varepsilon^{\dagger}(t)\right\rangle$, whose elements $r_{i j}$ are the second-order moments $r_{i j}=\left\langle\varepsilon_{i}(t) \varepsilon_{j}^{*}(t)\right\rangle \quad(i, j=1,2,3)$ of the zero-mean analytic signals $\varepsilon_{i}(t)$ of the respective components of the $3 \mathrm{D}$ instantaneous Jones vector $\boldsymbol{\varepsilon}(t)$ [13]. (The superscript ${ }^{\dagger}$ indicates conjugate transpose, * stands for complex conjugate, and the brackets indicate time averaging over the measurement time.) Thus $\mathbf{R}$ is a covariance matrix and therefore its three eigenvalues $\left(\lambda_{1}, \lambda_{2}, \lambda_{3}\right)$ are non-negative. The diagonal elements of $\mathbf{R}$ can be interpreted as the intensities associated with the respective $X Y Z$ components of the electric field, so that the quantity

$$
I=\operatorname{tr} \mathbf{R}=\left\langle\varepsilon_{1}^{2}(t)\right\rangle+\left\langle\varepsilon_{2}^{2}(t)\right\rangle+\left\langle\varepsilon_{3}^{2}(t)\right\rangle
$$

can be interpreted as the intensity of the whole state represented by $\mathbf{R}$. For an appropriate formulation of some later expressions, it will be useful to use the normalized form $\hat{\mathbf{R}} \equiv \mathbf{R} / I$ of the coherency matrix.

Let us now consider the Euclidean norm and the trace norm of $\mathbf{R}$, defined respectively as [13]

$$
\|\mathbf{R}\|_{2} \equiv \sqrt{\operatorname{tr}\left(\mathbf{R}^{2}\right)} ; \quad\|\mathbf{R}\|_{\mathrm{tr}} \equiv \operatorname{tr} \mathbf{R}=I,
$$

so that he $3 D$ degree of polarimetric purity $P_{(3)}$ [13] can be defined as $[1,2,4]$

$$
P_{(3)}=\sqrt{\frac{1}{2}\left(\frac{3\|\mathbf{R}\|_{2}^{2}}{\|\mathbf{R}\|_{\text {tr }}^{2}}-1\right) .}
$$

This invariant nondimensional quantity is limited to the interval $0 \leqslant P_{(3)} \leqslant 1$. The upper limit corresponds to the case that $\mathbf{R}$ has only one nonzero eigenvalue (pure state). 
Conversely, $P_{(3)}=0$ is reached when the three eigenvalues of $\mathbf{R}$ are equal (equiprobable mixture of states and zero correlation between the electric field components). Nevertheless, $P_{(3)}$ does not provide complete information about the polarimetric purity of a 3D state of polarization $\mathbf{R}$, and two quantities such as the two indices of polarimetric purity $[6,13,16,25]$ are required,

$$
P_{1}=\frac{\lambda_{1}-\lambda_{2}}{\operatorname{tr} \mathbf{R}}, \quad P_{2}=\frac{\lambda_{1}+\lambda_{2}-2 \lambda_{3}}{\operatorname{tr} \mathbf{R}},
$$

where $\lambda_{1}, \lambda_{2}, \lambda_{3}\left(\lambda_{1} \geqslant \lambda_{2} \geqslant \lambda_{3}\right)$ are the eigenvalues of $\mathbf{R}$. These invariant nondimensional parameters are restricted by the nested inequalities $0 \leqslant P_{1} \leqslant P_{2} \leqslant 1$.

Thus, while the pair $\left(P_{1}, P_{2}\right)$ provides detailed information of the structure of the polarimetric purity of $\mathbf{R}, P_{(3)}$ represents an overall measure of polarimetric purity, which can be calculated from $P_{1}$ and $P_{2}$ by the following weighted quadratic average [6,25]:

$$
P_{(3)}=\sqrt{3 P_{1}^{2}+P_{2}^{2}} / 2 .
$$

The physically feasible region in the purity space $\left(P_{1}, P_{2}\right)$ has been studied and interpreted by us in previous papers $[13,25]$, while interesting geometric representations for $\left(\lambda_{1}, \lambda_{2}, \lambda_{3}\right)$ and other derived quantities (including $P_{1}$ and $P_{2}$ ) have been presented by Sheppard [26,27]. The physical interpretation of $P_{1}, P_{2}$ and other parameters is considered below, as well as in the light of the case study performed in Sec. III, which, in turn, makes extensive use of the decompositions of a coherency matrix into convex sums of coherency matrices [13,31,43,44].

As follows from Ref. [32], $\mathbf{R}$ can be expressed as the following convex sum in terms of a set of $r$ (with $r \equiv \operatorname{rank} \mathbf{R}$ ) arbitrary independent 3D complex unit vectors $\mathbf{w}_{i}$ belonging to range $\mathbf{R}$ (i.e., belonging to the subspace generated by the eigenvectors of $\mathbf{R}$ with nonzero eigenvalues):

$$
\begin{aligned}
\mathbf{R} & =\sum_{i=1}^{r} p_{i} \mathbf{R}_{i}, \quad \mathbf{R}_{i} \equiv I\left(\mathbf{w}_{i} \otimes \mathbf{w}_{i}^{\dagger}\right), \\
p_{i} & =\frac{1}{I \sum_{j=1}^{r} \frac{1}{\lambda_{j}}\left|\left(\mathbf{U}^{\dagger} \mathbf{w}_{i}\right)_{j}\right|^{2}}, \quad \sum_{i=1}^{r} p_{i}=1, \quad I \equiv \operatorname{tr} \mathbf{R},
\end{aligned}
$$

where $\|$ indicates the modulus (or Euclidean norm), $\lambda_{i} \quad(i=1,2,3)$ are the ordered non-negative eigenvalues $\left(0 \leqslant \lambda_{3} \leqslant \lambda_{2} \leqslant \lambda_{1}\right)$ of $\mathbf{R}$, and $\mathbf{U}$ is the unitary matrix whose columns are the eigenvectors of $\mathbf{R}$. The arbitrary decomposition in Eq. (6) is formulated in the appropriate manner to emphasize the fact that the representatives of the components have been chosen to have the same intensity $I$, and consequently, $p_{i}+p_{2}+p_{3}=1$ (convex composition). Note that by taking as $\mathbf{w}_{i}$ the eigenvectors $\mathbf{u}_{i}(i=1,2,3)$ of $\mathbf{R}$, we get the spectral decomposition [13] of $\mathbf{R}$ as a particular case of the arbitrary decomposition.

Expansion (6) provides the way for generating arbitrary complete sets of the coherency matrices $I\left(\mathbf{w}_{i} \otimes \mathbf{w}_{i}^{\dagger}\right)$ of the pure components as well as their corresponding coefficients $p_{i}$. It should be noted that the number of pure components in the arbitrary decomposition is equal to $r$, thereby providing a meaningful physical interpretation of the integer parameter $r \equiv \operatorname{rank} \mathbf{R}$. Hereafter, when appropriate to indicate that a state $\mathbf{R}$ is pure, we will denote its coherence matrix as $\mathbf{R}_{p}$.

By considering the possible values of the indices of purity, we observe that they have a direct link with the purity structure of $\mathbf{R}$ and provide more detailed information than the very value of $r$. The following cases can be distinguished [25]: (1) when $0 \leqslant P_{1} \leqslant P_{2}<1(r=3), \mathbf{R}$ can be considered as composed of three pure states $\mathbf{R}=p_{1} \mathbf{R}_{p 1}+p_{2} \mathbf{R}_{p 2}+p_{3} \mathbf{R}_{p 3}$; if, in particular, $P_{2}=0$ (and hence, $P_{1}=0$ ), $\mathbf{R}$ is proportional to the identity matrix, $\mathbf{R}=I \operatorname{diag}(1,1,1) \equiv \mathbf{R}_{u-3 D}$, so that it represents a 3D unpolarized state (completely random polarization ellipse and completely random direction of propagation); (2) when $0 \leqslant P_{1}<P_{2}=1(r=2)$, $\mathbf{R}$ can be considered as composed of two pure states $\mathbf{R}=p_{1} \mathbf{R}_{p 1}+p_{2} \mathbf{R}_{p 2}$; if, in particular $P_{1}=0, \mathbf{R}$ corresponds to a two-dimensional (2D) unpolarized state propagating along a well-defined direction of propagation. (Note that due to the very definition of $\mathbf{R}$, it does not include the information about the sense of propagation of the wave.)

Finally, when $P_{1}=1$ (and hence $P_{2}=1$ and $r=1$ ), $\mathbf{R}$ corresponds to a pure state. While all the components of the arbitrary decomposition of $\mathbf{R}$ are pure states, it is also possible to decompose $\mathbf{R}$ by means of the characteristic (or trivial) decomposition [13]:

$$
\begin{aligned}
\mathbf{R} & =\mathbf{U d i a g}\left(\lambda_{1}, \lambda_{2}, \lambda_{3}\right) \mathbf{U}^{\dagger} \\
& =P_{1} I \hat{\mathbf{R}}_{1}+\left(P_{2}-P_{1}\right) I \hat{\mathbf{R}}_{2}+\left(1-P_{2}\right) I \hat{\mathbf{R}}_{3} ; \\
\hat{\mathbf{R}}_{1} & \equiv \mathbf{U d i a g}(1,0,0) \mathbf{U}^{\dagger}, \quad \hat{\mathbf{R}}_{2} \equiv \frac{1}{2} \mathbf{U d i a g}(1,1,0) \mathbf{U}^{\dagger}, \\
\hat{\mathbf{R}}_{3} & \equiv \frac{1}{3} \mathbf{U d i a g}(1,1,1) \mathbf{U}^{\dagger},
\end{aligned}
$$

where $\operatorname{rank} \hat{\mathbf{R}}_{1}=1, \operatorname{rank} \hat{\mathbf{R}}_{2}=2$, and $\operatorname{rank} \hat{\mathbf{R}}_{3}=3$, and all the components have been chosen to have the same intensity $I=$ $\operatorname{tr} \mathbf{R}$, so that the respective coefficients of the components are expressed in terms of the indices of polarimetric purity $\left(P_{1}, P_{2}\right)$.

The characteristic decomposition leads to a physical interpretation of $P_{1}$ as the ratio of power of the completely polarized part (or pure component) to the total power of the electromagnetic wave. Moreover, $P_{2}$ can be interpreted as the relative portion of power once the completely random component has been subtracted.

\section{THE INTRINSIC COHERENCY MATRIX}

The physically realizable rotations of the laboratory reference frame $X Y Z$ are represented by $3 \times 3$ orthogonal matrices $\mathbf{W}$ (with $\operatorname{det} \mathbf{W}=1$ ), so that the transformed coherency matrix $\mathbf{R}^{\prime}$ representing the same state as $\mathbf{R}$, but referred to the new reference frame, is given by $\mathbf{R}^{\prime}=\mathbf{W}^{T} \mathbf{R} \mathbf{W}$. Moreover, let us consider the decomposition of $\mathbf{R}$ into its real and imaginary parts $\mathbf{R}=\mathbf{R}_{R}+i \mathbf{R}_{I}$, where the real matrix $\mathbf{R}_{R} \equiv \operatorname{Re}(\mathbf{R})$ is symmetric and positive semidefinite, whereas the imaginary matrix $\mathbf{R}_{I} \equiv \operatorname{Im}(\mathbf{R})$ is skew symmetric $\left(\mathbf{R}_{I}=-\mathbf{R}_{I}^{T}\right)$. As Dennis pointed out [21], $\mathbf{R}_{R}$ can always be diagonalized through a particular rotation $\mathbf{Q}$ of the reference frame,

$$
\mathbf{Q}^{T} \mathbf{R}_{R} \mathbf{Q}=\operatorname{diag}\left(a_{1}, a_{2}, a_{3}\right) ; \quad\left(0 \leqslant a_{3} \leqslant a_{2} \leqslant a_{1}\right),
$$

so that $\mathbf{R}_{R}$ defines an ellipsoid (called by Dennis the inertia ellipsoid), whose semiaxes $\left(a_{1}, a_{2}, a_{3}\right)$ are aligned along the respective transformed axes $X_{O} Y_{O} Z_{O}$ (Fig. 1). Therefore, 


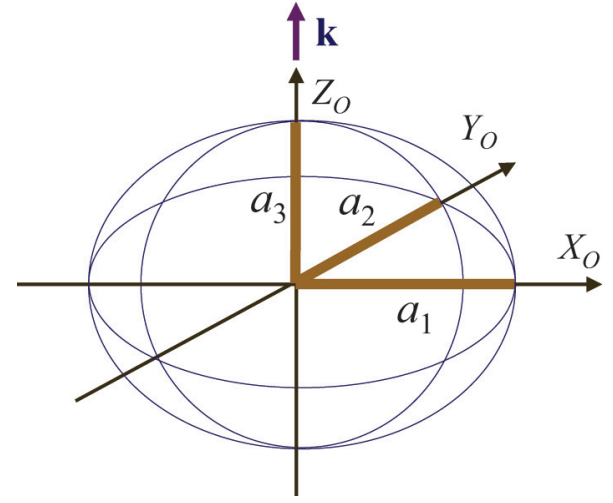

FIG. 1. (Color online) Intensity ellipsoid, with semiaxes $a_{1} \geqslant$ $a_{2} \geqslant a_{3}$, representing a mixed state constituted by the incoherent superposition of three pure linearly polarized states $\mathbf{R}_{p 1}, \mathbf{R}_{p 2}$, and $\mathbf{R}_{p 3}$. The direction $\mathbf{k}$ along the reference axis $Z_{O}$ can always be chosen as the common direction of propagation of the pure components $\mathbf{R}_{p 1}$ and $\mathbf{R}_{p 2}$ (with respective intensities $a_{1}, a_{2}$ ), whereas any direction orthogonal to $\mathbf{k}$ can be taken as the direction of propagation of the third pure component $\mathbf{R}_{p 3}$ (with intensity $a_{3}$ ).

$\operatorname{diag}\left(a_{1}, a_{2}, a_{3}\right)$ can be interpreted as the coherency matrix of a state composed of the incoherent superposition of three linearly polarized pure states,

$$
\begin{aligned}
\operatorname{diag}\left(a_{1}, a_{2}, a_{3}\right) & =\mathbf{R}_{p 1}+\mathbf{R}_{p 2}+\mathbf{R}_{p 3} \\
\mathbf{R}_{p 1} & \equiv a_{1} \operatorname{diag}(1,0,0), \quad \mathbf{R}_{p 2} \equiv a_{2} \operatorname{diag}(0,1,0) \\
\mathbf{R}_{p 3} & \equiv a_{3} \operatorname{diag}(0,0,1)
\end{aligned}
$$

with respective intensities $a_{1}, a_{2}$, and $a_{3}$. The previous decomposition is compatible with a variety of directions of propagation for each component. (The only condition is that the direction of propagation of each component is orthogonal to the respective polarization ellipse, degenerated into a straight segment along the respective axis $X_{O}, Y_{O}, Z_{O}$.) Since in particular $Z_{O}$ is orthogonal to the polarization axes of the states represented by $\mathbf{R}_{p 1}$ and $\mathbf{R}_{p 2}$, it is possible to choose the axis $Z_{O}$ as the common direction of propagation $\mathbf{k}$ for the pure states $\mathbf{R}_{p 1}$ and $\mathbf{R}_{p 2}$, while any axis orthogonal to $\mathbf{k}$ can be considered as the direction of propagation of the third pure component $\mathbf{R}_{p 3}$ (Fig. 1). We stress that this interpretation is not unique but is physically consistent, and, without any information additional to $\mathbf{R}$, is indistinguishable from other possible choices.

By applying the rotation $\mathbf{Q}$ to the whole matrix $\mathbf{R}$, we observe that the real and imaginary parts transform separately, so that we get the transformed coherency matrix [21]

$$
\begin{aligned}
\mathbf{R}_{O} & \equiv \mathbf{Q}^{T} \mathbf{R} \mathbf{Q} \\
& =\mathbf{Q}^{T} \mathbf{R}_{R} \mathbf{Q}+i \mathbf{Q}^{T} \mathbf{R}_{I} \mathbf{Q}=\operatorname{diag}\left(a_{1}, a_{2}, a_{3}\right)+i \mathbf{R}_{I}^{\prime}, \\
\mathbf{R}_{O} & \equiv\left[\begin{array}{ccc}
a_{1} & -i n_{3} & i n_{2} \\
i n_{3} & a_{2} & -i n_{1} \\
-i n_{2} & i n_{1} & a_{3}
\end{array}\right],
\end{aligned}
$$

where, as occurs for $\mathbf{R}_{I}, \mathbf{R}_{I}^{\prime}$ is real and skew symmetric. The orthogonal matrix $\mathbf{Q}$ can always be expressed as a product of rotations around the respective axes $Z X Y$ :

$$
\begin{aligned}
\mathbf{Q}= & \mathbf{Q}_{Z}(\varphi) \mathbf{Q}_{X}(\alpha) \mathbf{Q}_{Y}(\beta) \\
= & {\left[\begin{array}{ccc}
\cos \varphi & -\sin \varphi & 0 \\
\sin \varphi & \cos \varphi & 0 \\
0 & 0 & 1
\end{array}\right]\left[\begin{array}{ccc}
1 & 0 & 0 \\
0 & \cos \alpha & -\sin \alpha \\
0 & \sin \alpha & \cos \alpha
\end{array}\right] } \\
& \times\left[\begin{array}{ccc}
\cos \beta & 0 & -\sin \beta \\
0 & 1 & 0 \\
\sin \beta & 0 & \cos \beta
\end{array}\right] .
\end{aligned}
$$

Thus, since $\mathbf{R}=\mathbf{Q} \mathbf{R}_{O} \mathbf{Q}^{T}$, $\mathbf{R}$ can be parameterized in the form proposed by Dennis [21] through the following nine independent parameters: the three orientation angles $(\varphi, \alpha, \beta)$; the semiaxes of the intensity ellipsoid (or inertia ellipsoid [21]) given by the principal intensities $\left(a_{1}, a_{2}, a_{3}\right)$, and the three components $\left(n_{1}, n_{2}, n_{3}\right)$ of the angular momentum $\mathbf{n}$ of the wave along the respective axes $X_{O} Y_{O} Z_{O}$. In general, the orientation $\hat{\mathbf{n}} \equiv \mathbf{n} / \sqrt{n_{1}^{2}+n_{2}^{2}+n_{3}^{2}}$ of $\mathbf{n}$ differs from $\mathbf{k}$ [21]. The intensity $I$ of the state $\mathbf{R}_{O}$ is given by

$$
\begin{aligned}
I & =\operatorname{tr} \mathbf{R}=\lambda_{1}+\lambda_{2}+\lambda_{3}=\operatorname{tr} \mathbf{R}_{O}=\operatorname{tr} \mathbf{R}_{p 1}+\operatorname{tr} \mathbf{R}_{p 2}+\operatorname{tr} \mathbf{R}_{p 3} \\
& =a_{1}+a_{2}+a_{3} .
\end{aligned}
$$

Since the intrinsic coherency matrix $\mathbf{R}_{O}$ is positive semidefinite, the quantities $\left(a_{1}, a_{2}, a_{3} ; n_{1}, n_{2}, n_{3}\right)$ must satisfy the following set of constraining inequalities [21] derived from the non-negativity of the leading principal minors of $\mathbf{R}_{O}$ :

$$
\begin{gathered}
a_{1} \geqslant a_{2} \geqslant a_{3} \geqslant 0 ; \quad a_{1} a_{2} \geqslant n_{3}^{2}, \quad a_{1} a_{3} \geqslant n_{2}^{2}, \quad a_{2} a_{3} \geqslant n_{1}^{2} ; \\
a_{1} a_{2} a_{3} \geqslant a_{1} n_{1}^{2}+a_{2} n_{2}^{2}+a_{3} n_{3}^{2} .
\end{gathered}
$$

The smaller the third principal intensity $a_{3}$, the smaller the solid angle around the axis $Z_{O}$ that limits the range of compatible orientations of $\mathbf{n}$. When $a_{3}=0, \mathbf{n}$ is forced to lie along the axis $Z_{O}$, which, in turn, in this case is precisely the well-defined direction of propagation of the state $\mathbf{R}_{O}$.

Up to the limits set by the restrictive inequalities (13), the quantities $\left(a_{1}, a_{2}, a_{3} ; n_{1}, n_{2}, n_{3}\right)$ are independent and are intrinsic of a given coherency matrix $\mathbf{R}$. It should be stressed that the fact that the only physically realizable unitary transformations of the reference frame are those that are orthogonal leads to the indicated set of six physical parameters instead of the only three physical invariants derivable from the eigenvalues of $\mathbf{R}$. In other words, not all the unitary transformations of $\mathbf{R}$ are physically realizable as transformations of the laboratory reference frame, and consequently, a proper interpretation of the physical quantities involved in $\mathbf{R}$, as well as an appropriate analysis of the arbitrary and characteristic decompositions of $\mathbf{R}$, must be performed through orthogonal transformations (and hence exclude the unitary transformations that are not orthogonal). It should be noted that for some cases, transformations of the form $\mathbf{R}^{\prime}=\mathbf{V}^{T} \mathbf{R} \mathbf{V}, \mathbf{V}$ being a nonorthogonal unitary matrix, can be interpreted as the transformation of $\mathbf{R}$ due to the linear interaction with a certain nondiattenuating deterministic medium (as, for example, some kinds of birefringent phase plates) whose associated 3D Jones matrix is $\mathbf{V}$; but obviously, $\mathbf{R}^{\prime}$ corresponds to a state different than $\mathbf{R}$, and therefore this should not lead to confusion. 


\section{CASE ANALYSIS}

Once the arbitrary and characteristic decompositions, the structure of purity, and the orthogonal transformations of a generic $3 \times 3$ coherency matrix $\mathbf{R}$ have been considered, we are ready to undertake a study of the possible decompositions of a three-dimensional state of polarization represented by a given coherency matrix $\mathbf{R}$. To perform a proper case analysis, it is necessary to pay attention to the values of the integer parameters $r \equiv \operatorname{rank} \mathbf{R}=\operatorname{rank} \mathbf{R}_{O}$ and $t \equiv \operatorname{rank}[\operatorname{Re}(\mathbf{R})]=$ rank $\left[\operatorname{Re}\left(\mathbf{R}_{O}\right)\right]$. Concerning the physical meaning of these relevant parameters, we have observed that the arbitrary decomposition provides the interpretation of $r$ as the minimum number of pure arbitrary components of $\mathbf{R}$; moreover, $t$ is the number of nonzero components of the electric field in the intrinsic coherency matrix representation $\left(\mathbf{R}_{O}\right)$, i.e., the number of nonzero semiaxes of the intensity ellipsoid. As we will see in the following case analysis, $t=1$ corresponds to linearly polarized pure states, $t=2$ corresponds to states with a well-defined direction of propagation (2D states, excluding linearly polarized states), and $t=3$ corresponds to states that do not have a well-defined direction of propagation (excluding linearly polarized states).

\section{A. $\operatorname{Rank} R=1$} form

In this case, the intrinsic coherency matrix $\mathbf{R}_{O}$ takes the

$$
\mathbf{R}_{O}=\left[\begin{array}{ccc}
a_{1} & -i \sqrt{a_{1} a_{2}} & 0 \\
i \sqrt{a_{1} a_{2}} & a_{2} & 0 \\
0 & 0 & 0
\end{array}\right]
$$

so that the electric field lies in the transformed plane $X_{O} Y_{O}$. Now, by introducing the parameters $s_{0} \equiv a_{1}+a_{2}$ and $s_{3} \equiv 2 \sqrt{a_{1} a_{2}}$, together with the pair of parameters $s_{1} \equiv\left(a_{1}-a_{2}\right) \cos 2 \theta, s_{2} \equiv\left(a_{1}-a_{2}\right) \sin 2 \theta$, determined by the arbitrary choice of the angle $\theta$, we see that $\mathbf{R}_{O}$ can be expressed as [21]

$$
\mathbf{R}_{O}=\frac{1}{2}\left[\begin{array}{ccc}
s_{0}+\sqrt{s_{1}^{2}+s_{2}^{2}} & -i s_{3} & 0 \\
i s_{3} & s_{0}-\sqrt{s_{1}^{2}+s_{2}^{2}} & 0 \\
0 & 0 & 0
\end{array}\right],
$$

which corresponds to a $2 \mathrm{D}$ pure state propagating along the $Z_{O}$ axis. Furthermore, the polarization ellipse is oriented in such a manner that the major and minor semiaxes respectively lie in the new reference axes $X_{O}$ and $Y_{O}$. [Note that this is a result of the effect of the matrix $\mathbf{Q}_{Z}(\varphi)$ in the orthogonal transformation (12).] Thus an additional rotation of an arbitrary angle $\theta$ around the axis $Z_{O}$ provides the general expression of the coherency matrix $\mathbf{R}_{p Z_{O}}$ of a pure state propagating along the direction $Z_{O}$ in terms of the Stokes parameters $\left(s_{0}, s_{1}, s_{2}, s_{3}\right)$ (Fig. 2):

$$
\begin{aligned}
\mathbf{R}_{p Z_{O}} & \equiv \mathbf{Q}_{Z}(\theta) \mathbf{R}_{O} \mathbf{Q}_{Z}^{T}(\theta) \\
& \equiv \frac{1}{2}\left[\begin{array}{ccc}
s_{0}+s_{1} & s_{2}-i s_{3} & 0 \\
s_{2}+i s_{3} & s_{0}-s_{1} & 0 \\
0 & 0 & 0
\end{array}\right] ; \\
\cos 2 \theta & \equiv \frac{s_{1}}{\sqrt{s_{1}^{2}+s_{2}^{2}}}, \quad s_{0}=\sqrt{s_{1}^{2}+s_{2}^{2}+s_{3}^{2}} .
\end{aligned}
$$

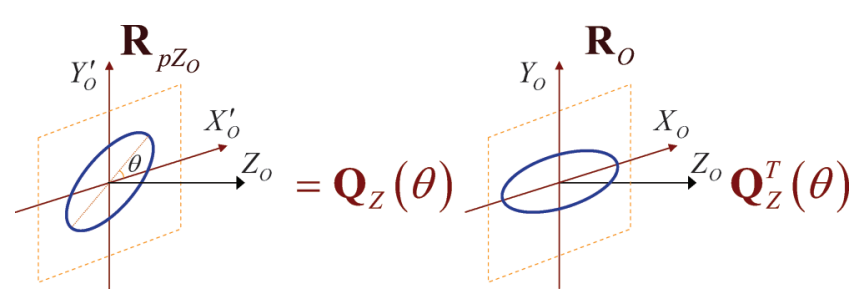

FIG. 2. (Color online) Coherency matrix $\mathbf{R}_{p Z_{O}}$ of a generic pure state propagating along the axis $Z_{O}$, obtained from its intrinsic coherency matrix $\mathbf{R}_{O}$ through an arbitrary rotation $\theta$ around $Z_{O}$.

In summary, the case $r=1$ corresponds to 2D pure states (i.e., totally polarized states). By considering now the two possible values of $t$ that are compatible with $r=1$, the following two subcases can be distinguished:

$$
\text { 1. } r=1, t=1,\left(a_{1}>0, a_{2}=a_{3}=0, n_{1}=n_{2}=n_{3}=0\right)
$$

$\mathbf{R}_{O}$ takes the simple form $\mathbf{R}_{O}=\operatorname{diag}\left(a_{1}, 0,0\right)$, where $a_{1}$ can be interpreted as the Stokes parameter $s_{1}$ of a linearly polarized state whose polarization ellipse degenerates into a segment along the axis $X_{O}$. It should be noted that in this case, $\mathbf{R}_{O}$ (and hence $\mathbf{R}$ ) is compatible with any direction of propagation perpendicular to the axis $X_{O}$. Obviously, under experimental conditions it is common to have specific complementary information about the direction and sense of propagation, but we stress that, in the case of a linearly polarized pure state, the very knowledge of the coherency matrix $\mathbf{R}$ does not determine the direction of propagation of the wave (Fig. 3). Furthermore, we also note that at the point in the space where $\mathbf{R}$ (and hence $\mathbf{R}_{O}$ ) is being considered, the incoherent superposition of a variety of pure states with linear polarizations along the $X_{O}$ axis, but with different directions of propagation, produces a pure state of linear polarization. Thus, without any further information than that provided by $\mathbf{R}$, this last case is polarimetrically indistinguishable from a pure state of linear

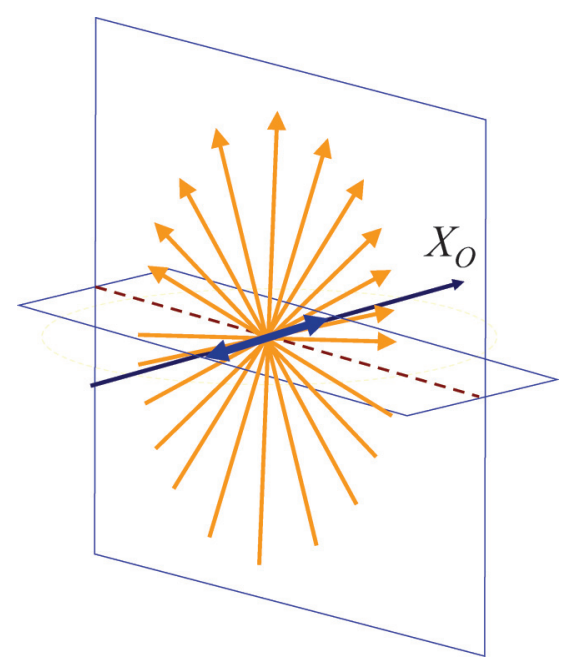

FIG. 3. (Color online) Representation of a linearly polarized pure state as the incoherent superposition of an arbitrary number of linearly polarized states with the same polarization axis along $X_{O}$ but with different directions of propagation perpendicular to $X_{O}$. 
polarization and fixed direction of propagation. The geometric nature of this peculiarity provides a method for superposing incoherently, at a fixed point in the space, a number of linearly polarized pure states whose arbitrary directions of propagation lie in a plane perpendicular to the common polarization axis $X_{O}$ and produce a polarimetrically pure state whose intensity is the sum of the intensities of the combined states.

\section{2. $r=1, t=2,\left(a_{1} \geqslant a_{2}>0, a_{3}=0, a_{1} a_{2}=n_{3}^{2}, n_{2}=n_{3}=0\right)$}

In this case, the polarization ellipse determines a plane $X_{O} Y_{O}$, so that the propagation direction $Z_{O}$ is orthogonal to the said plane and therefore it is well defined. Once the appropriate orthogonal transformation of the reference axes is performed, the case is reduced to a 2D pure state, with nonzero ellipticity, characterized by the coherency matrix

$$
\begin{aligned}
& \mathbf{R}_{p Z_{O}}=\mathbf{Q}_{Z}(\theta) \mathbf{R}_{O} \mathbf{Q}_{Z}^{T}(\theta)=\frac{1}{2}\left[\begin{array}{ccc}
s_{0}+s_{1} & s_{2}-i s_{3} & 0 \\
s_{2}+i s_{3} & s_{0}-s_{1} & 0 \\
0 & 0 & 0
\end{array}\right] ; \\
& \cos 2 \theta \equiv \frac{s_{1}}{\sqrt{s_{1}^{2}+s_{2}^{2}}}, \quad s_{0}=\sqrt{s_{1}^{2}+s_{2}^{2}+s_{3}^{2}} .
\end{aligned}
$$

\section{B. $\operatorname{Rank} R=2$}

The interpretation of the coherency matrix $\mathbf{R}$ with rank $\mathbf{R}=$ 2 depends substantially on the value of $t$. Since the value $t=1$ is not compatible with $r=2$, we distinguish the two possible cases $(r=2, t=2)$ and $(r=2, t=3)$.

$$
\text { 1. } r=2, t=2,\left(a_{1} \geqslant a_{2}>0, a_{3}=0, a_{1} a_{2}>n_{3}^{2}, n_{2}=n_{3}=0\right)
$$

It is straightforward to show that, similarly to the case $(r=1, t=2)$, the reference axes can be chosen in such a manner that the elements of the third row and of the third column of the coherency matrix are zero. The orthogonally transformed coherency matrix $\mathbf{R}_{Z_{O}}$ represents a partially polarized state with a well-defined direction of propagation $Z_{O}$, that is to say, a $2 \mathrm{D}$ partially polarized (or mixed) state:

$$
\mathbf{R}_{Z_{O}}=\mathbf{Q}_{Z}(\theta) \mathbf{R}_{O} \mathbf{Q}_{Z}^{T}(\theta)=\frac{1}{2}\left[\begin{array}{ccc}
s_{0}+s_{1} & s_{2}-i s_{3} & 0 \\
s_{2}+i s_{3} & s_{0}-s_{1} & 0 \\
0 & 0 & 0
\end{array}\right] ;
$$$$
\cos 2 \theta \equiv \frac{s_{1}}{\sqrt{s_{1}^{2}+s_{2}^{2}}}, \quad s_{0}>\sqrt{s_{1}^{2}+s_{2}^{2}+s_{3}^{2}} .
$$

Once the direction of propagation $Z_{O}$ has been determined, the state of polarization can be considered two-dimensional and described by a generic $2 \times 2$ coherency matrix,

$$
\begin{aligned}
\boldsymbol{\Phi}\left(I, P_{1}, \mathbf{u}\right)= & I \frac{1}{2}\left[\begin{array}{cc}
1+P_{1} u_{1} & P_{1}\left(u_{2}-i u_{3}\right) \\
P_{1}\left(u_{2}+i u_{3}\right) & 1-P_{1} u_{1}
\end{array}\right], \\
& \left(P_{1}<1\right),
\end{aligned}
$$

where the intensity $I=\operatorname{tr} \Phi$ is the time-averaged power density flux of the wave; $P_{1}$ (the first index of polarimetric purity) is the degree of polarization; and $u_{i}$ are the components of the unit vector $\mathbf{u}$ (Pauli axis) that summarizes the information about the azimuth $\varphi(0 \leqslant \varphi<\pi)$ and ellipticity angle $\chi$ $(-\pi / 4 \leqslant \chi \leqslant \pi / 4)$ of the average polarization ellipse

$$
\mathbf{u} \equiv\left(u_{1}, u_{2}, u_{3}\right)^{T}=(\cos 2 \chi \cos 2 \varphi, \cos 2 \chi \sin 2 \varphi, \sin 2 \chi)^{T} .
$$

The state $\boldsymbol{\Phi}\left(I, P_{1}, \mathbf{u}\right)$ can also be represented through the corresponding Stokes vector,

$$
\mathbf{s} \equiv\left(s_{0}, s_{1}, s_{2}, s_{3}\right)^{T}=I\left[\begin{array}{c}
1 \\
P_{1} \mathbf{u}
\end{array}\right], \quad\left(P_{1}<1\right) .
$$

a. Arbitrary decomposition. As it has been pointed out in previous works [10,13], a mixed 2D state $\boldsymbol{\Phi}$ can always be considered as an incoherent composition of two totally polarized (or pure) states $\boldsymbol{\Phi}_{p 1}$ and $\boldsymbol{\Phi}_{p 2}$. One of them can arbitrarily be chosen and then the second one is completely determined by this choice, so that there exist infinite possibilities for decomposing $\boldsymbol{\Phi}$ as a combination of two (or more) pure states:

$$
\begin{aligned}
\boldsymbol{\Phi}\left(I, P_{1}, \mathbf{u}\right) & =p \boldsymbol{\Phi}_{p 1}(I, \mathbf{v})+(1-p) \boldsymbol{\Phi}_{p 2}(I, \mathbf{w}) \\
\boldsymbol{\Phi}_{p 1}(I, \mathbf{v}) & \equiv I \frac{1}{2}\left[\begin{array}{cc}
1+v_{1} & v_{2}-i v_{3} \\
v_{2}+i v_{3} & 1-v_{1}
\end{array}\right], \\
\boldsymbol{\Phi}_{p 2}(I, \mathbf{w}) & \equiv I \frac{1}{2}\left[\begin{array}{cc}
1+w_{1} & w_{2}-i w_{3} \\
w_{2}+i w_{3} & 1-w_{1}
\end{array}\right], \\
p & =\frac{1-P_{1}^{2}}{2\left(1-P_{1} \mathbf{u}^{T} \mathbf{v}\right)}, \quad \mathbf{w}=\frac{P_{1} \mathbf{u}-p \mathbf{v}}{1-p} .
\end{aligned}
$$

A geometric view for the arbitrary decomposition of 2D states can be found in Ref. [10, p. 341].

Obviously, this arbitrary decomposition of $\boldsymbol{\Phi}$ can be considered either: (a) a convex combination of two pure states with equal intensities $I$, or (b) an additive combination of two pure states $\left[p \boldsymbol{\Phi}_{p 1}\right]$ and $\left[(1-p) \boldsymbol{\Phi}_{p 2}\right]$ with respective intensities $(p I)$ and $(I-p I)$. Despite the fact that both the said interpretations are equivalent and respectively physically realizable, it is particularly convenient to use the interpretation (a) because it is formulated in terms of representative states taken with the same trace norm $\|\boldsymbol{\Phi}\|_{\text {tr }}=I$ [32].

As a particular case of the above arbitrary decomposition, the choice $\mathbf{v}=\mathbf{u}$ leads to the well-known spectral decomposition of $\boldsymbol{\Phi}$ into two pure states represented by antipodal points on the Poincaré sphere:

$$
\boldsymbol{\Phi}\left(I, P_{1}, \mathbf{u}\right)=\frac{1+P_{1}}{2} \boldsymbol{\Phi}_{p}(I, \mathbf{u})+\frac{1-P_{1}}{2} \boldsymbol{\Phi}_{p}(I,-\mathbf{u}) .
$$

Arbitrary and spectral decompositions can be formulated in terms of Stokes vectors through the following respective expressions:

$$
\begin{aligned}
& \mathbf{s}=I\left[\begin{array}{c}
1 \\
P_{1} \mathbf{u}
\end{array}\right]=p I\left[\begin{array}{l}
1 \\
\mathbf{v}
\end{array}\right]+(1-p) I\left[\begin{array}{l}
1 \\
\mathbf{w}
\end{array}\right], \\
& \mathbf{s}=I\left[\begin{array}{c}
1 \\
P_{1} \mathbf{u}
\end{array}\right]=\frac{1+P_{1}}{2} I\left[\begin{array}{l}
1 \\
\mathbf{u}
\end{array}\right]+\frac{1-P_{1}}{2} I\left[\begin{array}{c}
1 \\
-\mathbf{u}
\end{array}\right] .
\end{aligned}
$$

We emphasize the potential applications of Eq. (24) in Stokes polarimetry (which has particular importance in several fields, such as, for instance, astronomic and atmospheric measurements [45]) because it provides a simple procedure 


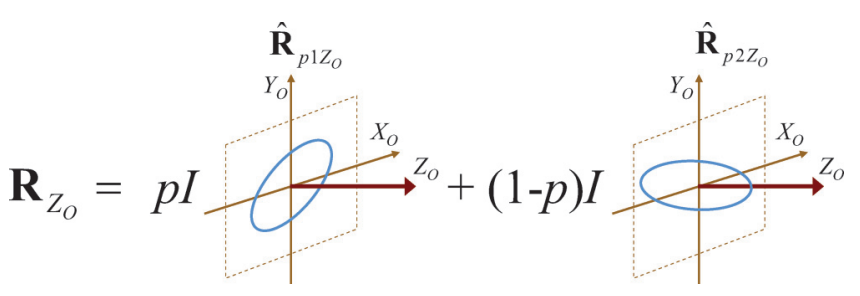

FIG. 4. (Color online) Arbitrary representation of a 2D mixed state (i.e., $r=2, t=2$ ) as the incoherent superposition of two pure states with the same propagation direction $Z_{O}$.

for the polarimetric subtraction of a reference Stokes vector from that obtained by experimental measurements [32].

In summary, the state $\mathbf{R}_{Z_{o}}$ corresponds to a $2 \mathrm{D}$ mixed state and is expressed as an incoherent superposition of two pure states with the same direction of propagation $Z_{O}$ (Fig. 4),

$$
\mathbf{R}_{Z_{o}}=p I \hat{\mathbf{R}}_{p 1 Z_{o}}+(1-p) I \hat{\mathbf{R}}_{p 2 Z_{o}},
$$

so that $\mathbf{R}_{Z_{O}}$ can be interpreted in terms of the following six independent parameters: two orientation angles that determine the common direction of propagation of the two components; intensity, azimuth, and ellipticity of the pure state $I \hat{\mathbf{R}}_{p 1 Z_{o}}$; and the coefficient $p$ of $I \hat{\mathbf{R}}_{p 1 Z_{o}}$ in the convex sum. Note that the intensity, azimuth, and ellipticity of $I \hat{\mathbf{R}}_{p 2 Z_{o}}$ are redundant parameters because they are derivable from the indicated set of six quantities.

b. Characteristic decomposition. An alternative interpretation of a 2D mixed state is achieved through the corresponding characteristic decomposition:

$$
\begin{aligned}
\boldsymbol{\Phi}\left(I, P_{1}, \mathbf{u}\right) & =P_{1} \boldsymbol{\Phi}_{p}(I, \mathbf{u})+\left(1-P_{1}\right) \boldsymbol{\Phi}_{u}(I), \\
\boldsymbol{\Phi}_{u} & \equiv I \frac{1}{2}\left[\begin{array}{ll}
1 & 0 \\
0 & 1
\end{array}\right] ; \\
\mathbf{s} & =I\left[\begin{array}{c}
1 \\
P_{1} \mathbf{u}
\end{array}\right]=P_{1} I\left[\begin{array}{l}
1 \\
\mathbf{u}
\end{array}\right]+\left(1-P_{1}\right) I\left[\begin{array}{l}
1 \\
\mathbf{0}
\end{array}\right],
\end{aligned}
$$

where $\boldsymbol{\Phi}_{p}$ represents a 2D pure state and $\boldsymbol{\Phi}_{u}$ represents a 2D unpolarized state, both propagating along the same direction $Z_{O}$. This is the well-known $2 \mathrm{D}$ form of the characteristic decomposition.

Returning to the $3 \mathrm{D}$ representation, the characteristic decomposition is expressed as

$$
\begin{aligned}
\mathbf{R}_{Z_{O}} & =P_{1} \mathbf{R}_{p Z_{o}}+\left(1-P_{1}\right) \mathbf{R}_{u-2 D} \\
\mathbf{R}_{p Z_{O}} & \equiv I \frac{1}{2}\left[\begin{array}{ccc}
1+u_{1} & u_{2}-i u_{3} & 0 \\
u_{2}+i u_{3} & 1-u_{1} & 0 \\
0 & 0 & 0
\end{array}\right], \\
\mathbf{R}_{u-2 D} & \equiv I \frac{1}{2}\left[\begin{array}{lll}
1 & 0 & 0 \\
0 & 1 & 0 \\
0 & 0 & 0
\end{array}\right],
\end{aligned}
$$

where $\mathbf{R}_{p Z_{o}}$ represents a pure state and $\mathbf{R}_{u-2 D}$ represents a $2 \mathrm{D}$ unpolarized state, both propagating along the same direction $Z_{O}$. Once the laboratory reference axes have been appropriately rotated, the characteristic decomposition of a state $\mathbf{R}$ with $r=2, t=2$ is expressed as that of a generic 2D state [Eq. (26)]. Thus in the case of $r=2, t=2$, the characteristic decomposition leads to the following interpretation of

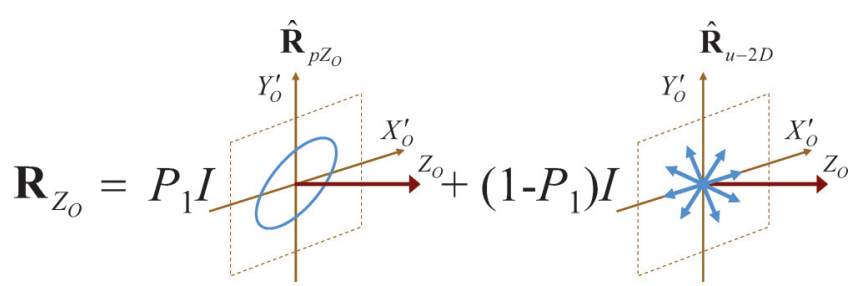

FIG. 5. (Color online) Characteristic representation of a 2D mixed state (i.e., $r=2, t=2$ ) as the incoherent superposition of a pure state and a $2 \mathrm{D}$ unpolarized state with the same propagation direction $Z_{O} . P_{1}$ is the first index of polarimetric purity (or degree of polarization).

the coherency matrix in terms of six independent parameters (Fig. 5): two orientation angles that determine the direction of propagation of the pure component $\mathbf{R}_{p Z_{o}}$; intensity, azimuth, and ellipticity of the pure component $\mathbf{R}_{p Z_{o}}$; and the degree of polarization $P_{1}$ of $\mathbf{R}_{Z_{o}}$, which is the coefficient of $\mathbf{R}_{p Z_{o}}$ in the convex sum.

$$
\begin{aligned}
& \text { 2. } r=2, t=3,\left(a_{1} \geqslant a_{2} \geqslant a_{3}>0\right. \text {; } \\
& \left.a_{1} a_{2} a_{3}=a_{1} n_{1}^{2}+a_{2} n_{2}^{2}+a_{3} n_{3}^{2}\right)
\end{aligned}
$$

When $t=3$, the electric field $\boldsymbol{E}$ of the electromagnetic wave has necessarily three nonzero orthogonal components, so that $\boldsymbol{E}$ does not evolve inside a fixed plane, and thus the direction of propagation is not well defined. The analogy with the $2 \mathrm{D}$ representation is no longer applicable for the state represented by $\mathbf{R}$.

a. Arbitrary decomposition. Any pure state belonging to range $\mathbf{R}$ can be considered as a component (note that range $\mathbf{R}_{O}=$ range $\mathbf{R}$ ), and the second pure component as well as the respective coefficients are straightforwardly determined. Thus the arbitrary decomposition $\mathbf{R}=p I \hat{\mathbf{R}}_{p 1}+$ $(1-p) I \hat{\mathbf{R}}_{p 2}$ (Fig. 6) can be performed either by taking a set of two independent complex unit vectors $\left(\mathbf{w}_{1}, \mathbf{w}_{2}\right)$ belonging to range $\mathbf{R}$ as the generators of the components $\hat{\mathbf{R}}_{p 1}=\mathbf{w}_{1} \times \mathbf{w}_{1}^{\dagger}$ and $\hat{\mathbf{R}}_{p 2}=\mathbf{w}_{2} \times \mathbf{w}_{2}^{\dagger}$ and calculating the coefficient $p$ by means of Eq. (6), or through the following procedure [13]:

(i) Take an arbitrary $3 \mathrm{D}$ complex unit vector $\mathbf{w}_{1}$ belonging to range $\mathbf{R}$, and synthesize the normalized coherency matrix $\hat{\mathbf{R}}_{p 1} \equiv \mathbf{w}_{1} \times \mathbf{w}_{1}^{\dagger}$ of the first component;

(ii) Calculate the coefficient $p$ of $\hat{\mathbf{R}}_{p 1}: p=$ $\left\{\operatorname{tr}\left[\operatorname{diag}\left(\lambda_{1}, \lambda_{2}, 0\right) \hat{\mathbf{R}}_{p 1}\right]\right\} / I ; \quad\left(I \equiv \lambda_{1}+\lambda_{2}\right) ;$

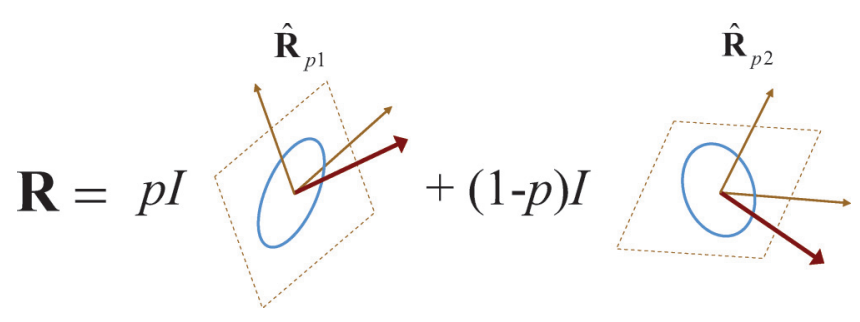

FIG. 6. (Color online) Arbitrary representation of a mixed state $\mathbf{R}$ with rank $\mathbf{R}=2$ and $\operatorname{rank}[\operatorname{Re}(\mathbf{R})]=3$ as the incoherent superposition of two pure states with different directions of propagation. In spite of rank $\mathbf{R}=2, \mathbf{R}$ corresponds to a $3 \mathrm{D}$ mixed state. 
(iii) And calculate the second pure component: $\hat{\mathbf{R}}_{p 2}=$ $\left(\hat{\mathbf{R}}-p \hat{\mathbf{R}}_{p 1}\right) /(1-p)$.

Obviously, the previous procedure can be applied either to $\mathbf{R}$ or to $\mathbf{R}_{O}$, depending on the reference frame considered.

Since any superposition of pure states with the same direction of propagation is represented by a coherency matrix $\mathbf{R}_{O}$ with $a_{3}=0$, we conclude that when $r=2$ and $t=3$, the state $\mathbf{R}$ can be considered as the superposition of two pure states whose propagation directions are different. In other words, the previous analysis demonstrates the surprising result that a coherency matrix $\mathbf{R}$ with $\operatorname{rank} \mathbf{R}=2$ and $\operatorname{rank}[\operatorname{Re}(\mathbf{R})]=3$ cannot be synthesized through the superposition of two pure states propagating along the same direction. We stress that this result contradicts the commonly accepted idea that coherency matrices with rank $\mathbf{R}=2$ necessarily correspond to $2 \mathrm{D}$ states (i.e., with a well-defined direction of propagation).

Consequently, in the case of $r=2, t=3$, the coherency matrix can be interpreted in terms of the following set of eight independent parameters: two orientation angles that determine the direction of propagation of the first component $I \hat{\mathbf{R}}_{p 1}$ of the arbitrary decomposition; intensity, azimuth, and ellipticity of the pure state $I \hat{\mathbf{R}}_{p 1}$; two orientation angles that determine the direction of propagation of the second pure component $I \hat{\mathbf{R}}_{p 2}$ of the arbitrary decomposition; and the coefficient $p$ of the convex sum in the arbitrary decomposition of $\mathbf{R}$. Note that the intensity, azimuth, and ellipticity of $I \hat{\mathbf{R}}_{p 2}$ are redundant parameters because they are derivable from the indicated set of eight quantities.

b. Characteristic decomposition. In this case, the characteristic decomposition of $\mathbf{R}$ is formulated as follows:

$$
\begin{gathered}
\mathbf{R}=\mathbf{U}\left[\begin{array}{ccc}
\lambda_{1} & 0 & 0 \\
0 & \lambda_{2} & 0 \\
0 & 0 & 0
\end{array}\right] \mathbf{U}^{\dagger}=P_{1} I \hat{\mathbf{R}}_{p}+\left(1-P_{1}\right) I \hat{\mathbf{R}}_{m} ; \\
\hat{\mathbf{R}}_{p} \equiv \mathbf{U}\left[\begin{array}{lll}
1 & 0 & 0 \\
0 & 0 & 0 \\
0 & 0 & 0
\end{array}\right] \mathbf{U}^{\dagger}, \hat{\mathbf{R}}_{m} \equiv \frac{1}{2} \mathbf{U}\left[\begin{array}{lll}
1 & 0 & 0 \\
0 & 1 & 0 \\
0 & 0 & 0
\end{array}\right] \mathbf{U}^{\dagger},
\end{gathered}
$$

in terms of the pure component $\hat{\mathbf{R}}_{p}$ and the nonpure component $\hat{\mathbf{R}}_{m}$. While $\hat{\mathbf{R}}_{p}$ has an immediate physical interpretation as a completely polarized state, the physical interpretation of $\hat{\mathbf{R}}_{m}$ requires the consideration of the value of the integer parameter $r_{m} \equiv \operatorname{rank}\left[\operatorname{Re}\left(\hat{\mathbf{R}}_{m}\right)\right]:$

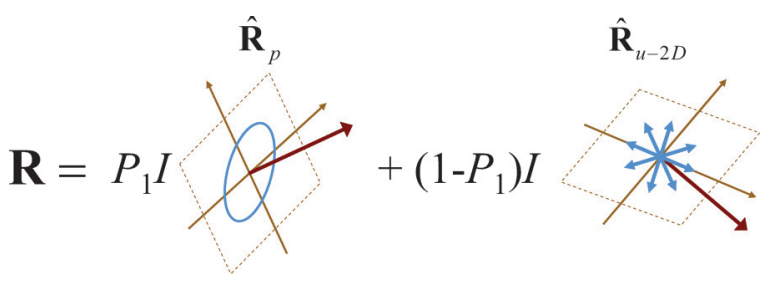

FIG. 7. (Color online) Characteristic representation of a mixed state $\mathbf{R}$ with $\operatorname{rank} \mathbf{R}=2, \operatorname{rank}[\operatorname{Re}(\mathbf{R})]=3$ and $\operatorname{rank}\left[\operatorname{Re}\left(\mathbf{R}_{m}\right)\right]=2$ as the incoherent superposition of a pure state and a $2 \mathrm{D}$ unpolarized state, with different directions of propagation. In spite of rank $\mathbf{R}=2$, $\mathbf{R}$ corresponds to a 3D mixed state.

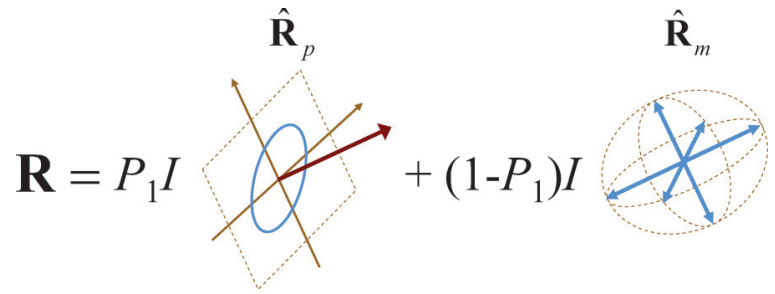

FIG. 8. (Color online) Characteristic representation of a mixed state $\mathbf{R}$ with rank $\mathbf{R}=2$, $\operatorname{rank}[\operatorname{Re}(\mathbf{R})]=3$ and $\operatorname{rank}\left[\operatorname{Re}\left(\mathbf{R}_{m}\right)\right]=3$ as the incoherent superposition of a pure state and a $3 \mathrm{D}$ mixed state. In spite of rank $\mathbf{R}=2, \mathbf{R}$ corresponds to a $3 \mathrm{D}$ mixed state.

(i) When $r_{m}=2, \hat{\mathbf{R}}_{m}$ represents a $2 \mathrm{D}$ unpolarized state $\hat{\mathbf{R}}_{u-2 D}$ propagating along a direction different from that of $\hat{\mathbf{R}}_{p}$, and therefore $\mathbf{R}$ can be interpreted by means of the following eight parameters provided by its characteristic decomposition (Fig. 7): two orientation angles that determine the direction of propagation of the pure component $\mathbf{R}_{p} \equiv I \hat{\mathbf{R}}_{p}$; intensity, azimuth, and ellipticity of the pure component $\mathbf{R}_{p}$; two orientation angles that determine the direction of propagation of the 2 D unpolarized component $\mathbf{R}_{m} \equiv I \hat{\mathbf{R}}_{u-2 D}$; and the first index of polarimetric purity (or degree of polarization) $P_{1}$ of $\mathbf{R}$, which is the coefficient of $\mathbf{R}_{p}$ in the convex sum. (We recall that when $r<3$, the second index of polarimetric purity $P_{2}$ is equal to 1 , and thus the coefficient $P_{2}-P_{1}$ of $\mathbf{R}_{m}$ becomes $1-P_{1}$.)

(ii) When $r_{m}=3, \mathbf{R}_{m}$ represents a 3D partially polarized (but not totally random) state without a well-defined direction of propagation. From the characteristic decomposition, $\mathbf{R}$ can be interpreted by means of the following eight parameters (Fig. 8): two orientation angles that determine the direction of propagation of the pure component $\mathbf{R}_{p}$; the azimuth and the ellipticity of the pure component $\mathbf{R}_{p}$; the principal intensities $\left(m_{1}, m_{2}, m_{3}\right)$ of $\mathbf{R}_{m}$, i.e., the eigenvalues of $\operatorname{Re}\left(\mathbf{R}_{m}\right)$ (note that the intensity $I$ is obtainable as $\left.I=\operatorname{tr} \mathbf{R}_{m}=m_{1}+m_{2}+m_{3}\right)$; and the first index of polarimetric purity (or degree of polarization) $P_{1}$ of $\mathbf{R}$.

$$
\begin{gathered}
\text { C. } \operatorname{Rank} \mathrm{R}=3 \\
\left(a_{1} \geqslant a_{2} \geqslant a_{3}>\mathbf{0}, \quad a_{1} a_{2} a_{3}>a_{1} n_{1}^{2}+a_{2} n_{2}^{2}+a_{3} n_{3}^{2}\right)
\end{gathered}
$$

In this case, the only achievable value of $t \equiv \operatorname{rank}[\operatorname{Re}(\mathbf{R})]$ is $t=3$, so that the electric field $\boldsymbol{E}$ of the electromagnetic wave has necessarily three nonzero orthogonal components, and thus the direction of propagation is not well defined. As in the previous case, in order to get appropriate physical interpretation, let us consider separately the arbitrary and characteristic decompositions.

\section{Arbitrary decomposition}

When rank $\mathbf{R}=3$, the arbitrary decomposition of $\mathbf{R}$ can be performed either by taking a set of three independent complex unit vectors $\left(\mathbf{w}_{1}, \mathbf{w}_{2}, \mathbf{w}_{3}\right)$ as the generators of the respective arbitrary components and calculating the coefficients by means of Eq. (6); or through the following procedure [13]:

(i) Take an arbitrary $3 \mathrm{D}$ complex unit vector $\mathbf{w}_{1}$ (note that $\mathbf{w}_{1}$ necessarily belongs to range $\mathbf{R}$, because range $\mathbf{R}$ covers the 
complete 3D complex space) and synthesize the normalized coherency matrix $\hat{\mathbf{R}}_{p 1} \equiv \mathbf{w}_{1} \times \mathbf{w}_{1}^{\dagger}$ of the first component;

(ii) Calculate the coefficient $p_{1}$ of $\hat{\mathbf{R}}_{p 1}$ :

$$
p_{1}=\frac{1}{I}\left\{\operatorname{tr}\left[\operatorname{diag}\left(\lambda_{1}, \lambda_{2}, \lambda_{3}\right) \hat{\mathbf{R}}_{p 1}\right]\right\} ; \quad\left(I \equiv \lambda_{1}+\lambda_{2}+\lambda_{3}\right)
$$

(iii) Calculate the remainder coherency matrix $\hat{\mathbf{R}}_{r}=$ $\left[\hat{\mathbf{R}}-p_{1}\left(\mathbf{w}_{1} \times \mathbf{w}_{1}^{\dagger}\right)\right] /\left(1-p_{1}\right)$

(iv) Take an arbitrary $3 \mathrm{D}$ complex unit vector $\mathbf{w}_{2}$ belonging to range $\mathbf{R}_{r}$ and synthesize the normalized coherency matrix $\hat{\mathbf{R}}_{p 2} \equiv \mathbf{w}_{2} \times \mathbf{w}_{2}^{\dagger}$ of the second component;

(v) Calculate the coefficient $p_{2}$ of $\hat{\mathbf{R}}_{p 2}$ in the convex sum

$$
p_{2}=\frac{1}{I} \operatorname{tr}\left\{\operatorname{diag}\left(\lambda_{1}, \lambda_{2}, \lambda_{3}\right)\left[\mathbf{w}_{2} \otimes \mathbf{w}_{2}^{\dagger}\right]\right\}
$$

(vi) And calculate the third pure component and its coefficient through the expressions:

$$
\begin{aligned}
\hat{\mathbf{R}}_{p 3} & =\frac{\hat{\mathbf{R}}-p_{1}\left(\mathbf{w}_{1} \times \mathbf{w}_{1}^{\dagger}\right)-p_{2}\left(\mathbf{w}_{2} \times \mathbf{w}_{2}^{\dagger}\right)}{\left(1-p_{1}-p_{2}\right)} \\
p_{3} & =1-p_{1}-p_{2} .
\end{aligned}
$$

With respect to the directions of propagation of the three pure components of the arbitrary decomposition, there are two possibilities, which depend on the particular choices of the components: (1) two components have the same direction of propagation but different to that of the remainder component, and (2) the three pure components have different directions of propagation.

The arbitrary decomposition of $\mathbf{R}$ leads to its interpretation in terms of the following nine independent parameters (Fig. 9): two orientation angles that determine the direction of propagation of the first component $\mathbf{R}_{p 1} \equiv I \hat{\mathbf{R}}_{p 1}$; intensity, azimuth, and ellipticity of the pure state $\mathbf{R}_{p 1}$; two orientation angles that determine the direction of propagation of the pure component $\mathbf{R}_{p 2}$ (or, if the said direction coincides with the direction of propagation of $\mathbf{R}_{p 1}$, the orientation angles that determine the direction of propagation of $\mathbf{R}_{p 3}$ ); and the coefficients $p_{1}$ and $p_{2}$ (recall that $p_{3}=1-p_{1}-p_{2}$ ). Note that other parameters of the equivalent system, such as the intensity, azimuth, and ellipticity of $\mathbf{R}_{p 2}$ and of $\mathbf{R}_{p 3}$, are redundant because they are derivable from the indicated set of nine quantities.

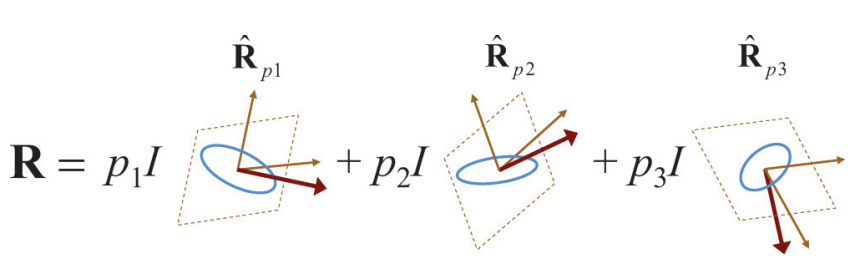

FIG. 9. (Color online) Arbitrary representation of a mixed state $\mathbf{R}$ with rank $\mathbf{R}=3$ as the incoherent superposition of three pure states with different directions of propagation.

\section{Characteristic decomposition}

When rank $\mathbf{R}=3$, the characteristic decomposition of $\mathbf{R}$ has the general form

$$
\begin{aligned}
& \mathbf{R}= \mathbf{U}\left[\begin{array}{ccc}
\lambda_{1} & 0 & 0 \\
0 & \lambda_{2} & 0 \\
0 & 0 & \lambda_{3}
\end{array}\right] \mathbf{U}^{\dagger}=P_{1} I \hat{\mathbf{R}}_{p}+\left(P_{2}-P_{1}\right) I \hat{\mathbf{R}}_{m} \\
&+\left(1-P_{2}\right) I \hat{\mathbf{R}}_{u-3 D} ; \\
& \hat{\mathbf{R}}_{p} \equiv \mathbf{U}\left[\begin{array}{lll}
1 & 0 & 0 \\
0 & 0 & 0 \\
0 & 0 & 0
\end{array}\right] \mathbf{U}^{\dagger}, \quad \hat{\mathbf{R}}_{m} \equiv \frac{1}{2} \mathbf{U}\left[\begin{array}{ccc}
1 & 0 & 0 \\
0 & 1 & 0 \\
0 & 0 & 0
\end{array}\right] \mathbf{U}^{\dagger}, \\
& \hat{\mathbf{R}}_{u-3 D} \equiv \frac{1}{3}\left[\begin{array}{lll}
1 & 0 & 0 \\
0 & 1 & 0 \\
0 & 0 & 1
\end{array}\right],
\end{aligned}
$$

where $\hat{\mathbf{R}}_{p}$ represents a pure state and $\hat{\mathbf{R}}_{u-3 D}$ represents a $3 \mathrm{D}$ unpolarized state. The characteristic decomposition is completed with the second component $I \hat{\mathbf{R}}_{m}$, whose interpretation can be performed as follows in terms of the value of the auxiliary parameter $r_{m} \equiv \operatorname{rank}\left[\operatorname{Re}\left(\hat{\mathbf{R}}_{m}\right)\right]$.

(i) When $r_{m}=2, \hat{\mathbf{R}}_{m}$ represents a $2 \mathrm{D}$ unpolarized state $\hat{\mathbf{R}}_{u-2 D}$ propagating along a direction different than that of $\hat{\mathbf{R}}_{p}$. Thus $\mathbf{R}$ can be expressed as

$$
\begin{aligned}
\mathbf{R}= & P_{1} I \hat{\mathbf{R}}_{p}+\left(P_{2}-P_{1}\right) I \hat{\mathbf{R}}_{u-2 D} \\
& +\left(1-P_{2}\right) I \hat{\mathbf{R}}_{u-3 D},
\end{aligned}
$$

and therefore, $\mathbf{R}$ can be interpreted by means of the following nine independent parameters (Fig. 10): two orientation angles that determine the direction of propagation of the pure component $\mathbf{R}_{p} \equiv I \hat{\mathbf{R}}_{p}$; intensity, azimuth, and ellipticity of the pure component $\mathbf{R}_{p}$; two orientation angles that determine the direction of propagation of the $2 \mathrm{D}$ unpolarized component $\mathbf{R}_{m} \equiv I \hat{\mathbf{R}}_{u-2 D}$; and the two indices of polarimetric purity $\left(P_{1}, P_{2}\right)$ of $\mathbf{R}$.

(ii) When $r_{m}=3$, the components $\mathbf{R}_{p}$ and $\mathbf{R}_{m}$ can be recombined and redecomposed in such a manner that the characteristic decomposition of $\mathbf{R}$ can be rewritten in the following appropriate form:

$$
\begin{aligned}
& \mathbf{R}= \frac{P_{1}+P_{2}}{2} I \hat{\mathbf{R}}_{p 1}+\frac{P_{2}-P_{1}}{2} \boldsymbol{I} \hat{\mathbf{R}}_{p 2} \\
&+\left(1-P_{2}\right) I \hat{\mathbf{R}}_{u-3 D} ; \\
& \hat{\mathbf{R}}_{p 1} \equiv \mathbf{U}\left[\begin{array}{lll}
1 & 0 & 0 \\
0 & 0 & 0 \\
0 & 0 & 0
\end{array}\right] \mathbf{U}^{\dagger},
\end{aligned}
$$

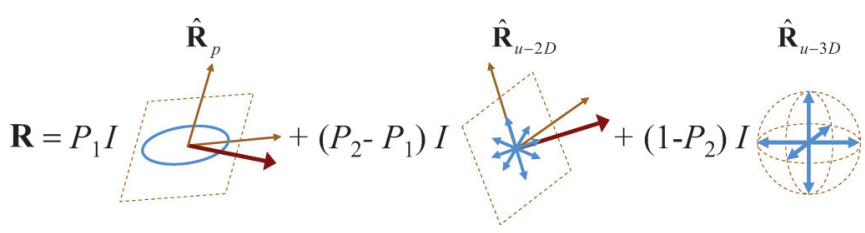

FIG. 10. (Color online) Characteristic representation of a mixed state $\mathbf{R}$ with $\operatorname{rank} \mathbf{R}=3$, and $\operatorname{rank}\left[\operatorname{Re}\left(\mathbf{R}_{m}\right)\right]=2$ as the incoherent superposition of a pure state, a 2D unpolarized state (with different direction of propagation), and a 3D unpolarized state. 


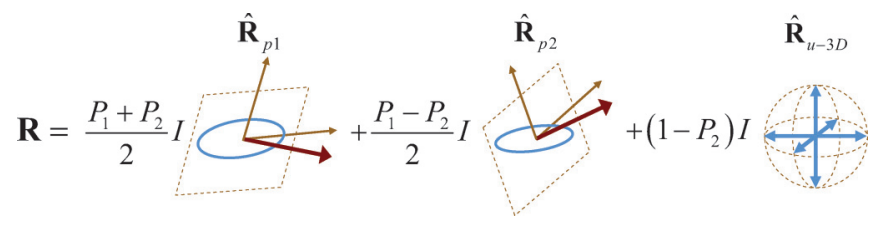

FIG. 11. (Color online) Characteristic representation of a mixed state $\mathbf{R}$ with $\operatorname{rank} \mathbf{R}=3$, and $\operatorname{rank}\left[\operatorname{Re}\left(\mathbf{R}_{m}\right)\right]=3$ as the incoherent superposition of two pure states (with different directions of propagation) and a 3D unpolarized state.

$$
\begin{aligned}
\hat{\mathbf{R}}_{p 2} & \equiv \mathbf{U}\left[\begin{array}{lll}
0 & 0 & 0 \\
0 & 1 & 0 \\
0 & 0 & 0
\end{array}\right] \mathbf{U}^{\dagger}, \\
\hat{\mathbf{R}}_{u-3 D} & \equiv \frac{1}{3}\left[\begin{array}{lll}
1 & 0 & 0 \\
0 & 1 & 0 \\
0 & 0 & 1
\end{array}\right],
\end{aligned}
$$

where the two first components are pure states with different directions of propagation and $\hat{\mathbf{R}}_{u-3 D}$ is a $3 \mathrm{D}$ unpolarized state. Thus $\mathbf{R}$ can be interpreted by means of the following nine independent parameters (Fig. 11): two orientation angles that determine the direction of propagation of the first pure component $\mathbf{R}_{p 1} \equiv I \hat{\mathbf{R}}_{p 1}$; intensity, azimuth, and ellipticity of $\mathbf{R}_{p 1}$; two orientation angles that determine the direction of propagation of the second pure component $\mathbf{R}_{p 2}$; and the two indices of purity $\left(P_{1}, P_{2}\right)$ of $\mathbf{R}$. Note that the azimuth and ellipticity of $\mathbf{R}_{p 2} \equiv I \hat{\mathbf{R}}_{p 2}$ are redundant because they are derivable from the indicated set of nine parameters.

\section{THE DEGREE OF DIRECTIONALITY}

As it has been pointed out above, the 3D coherency matrix $\mathbf{R}$ is defined for a given fixed point $\mathbf{r}$ in the space and does not contain direct information about the direction of propagation of the electromagnetic wave. Nevertheless, in the previous section we have found that when the rank of the real part of the coherency matrix is equal to $2(t \equiv \operatorname{rank}[\operatorname{Re}(\mathbf{R})]=2)$, the evolution of the electric field defines a fixed plane $\Pi$, and consequently the direction $\mathbf{k}$ perpendicular to $\Pi$, and hence perpendicular to the wave front, can properly be considered as the direction of propagation at point $\mathbf{r}$.

Moreover, when $t=3$, the evolution of the electric field of the wave defines the intensity ellipsoid with three nonzero semiaxes. Except for the case of 3D unpolarized states (whose intensity ellipsoid is a sphere), the direction $\mathbf{k}$ perpendicular to the plane of symmetry containing the maximum ellipse (Fig. 1), i.e., along the direction defined by the axis $Z_{0}$, can be considered as the average direction of propagation.

Thus it is highly desirable to define a measure of the directional purity of the state $\mathbf{R}$, i.e., a measure of the degree of dominance (or stability) of the average direction of propagation (total dominance for states with $t=2$, and zero dominance for 3D unpolarized states). As a previous attempt to get a definition of such a degree of directionality, we proposed the use of the second index of polarimetric purity $P_{2}$, but the analysis performed in the previous sections shows that when $r \equiv \operatorname{rank} \mathbf{R}=2$ and $t=3$, then $P_{2}=1$, despite the fact that the direction of propagation is not well defined. Consequently, in general $P_{2}$ does not constitute an appropriate measure of the degree of directionality.

In light of the physical interpretation of the intrinsic coherency matrix $\mathbf{R}_{O}(\mathbf{R})$ and inspired by the definition of $P_{2}$ in terms of the eigenvalues of $\mathbf{R}$, we find that a proper candidate for the desired degree of directionality is given by the nondimensional quantity defined as

$$
P_{d} \equiv \frac{a_{1}+a_{2}-2 a_{3}}{a_{1}+a_{2}+a_{3}},
$$

where, as indicated in Eq. (8), the real non-negative quantities $\left(a_{1}, a_{2}, a_{3}\right)$ are the eigenvalues of $\operatorname{Re}(\mathbf{R})$, with the choice $0 \leqslant a_{3} \leqslant a_{2} \leqslant a_{1}$. The appropriateness of this definition of $P_{d}$ is justified from the fact that this parameter reaches its maximum value $P_{d}=1$ for $a_{3}=0$ (absence of uncertainty in the direction of propagation), whereas $P_{d}=0$ corresponds exclusively to a $3 \mathrm{D}$ unpolarized state $\mathbf{R}_{u-3 D}$, whose associated direction of propagation is completely random. In agreement with the case study performed in Sec. III, intermediate cases have appropriate and consistent values in the interval $0<P_{d}<1$.

It should be noted that in the particular case of linearly polarized pure states, $\operatorname{Re}\left(\mathbf{R}_{O}\right)=\operatorname{diag}\left(a_{1}, 0,0\right)$ has the peculiarity that it is compatible with any direction of propagation that is orthogonal to the axis $X_{O}$ (Fig. 1) and even can be considered as an incoherent superposition of an arbitrary set of linearly polarized pure states propagating along different directions (provided that such directions lie in the plane perpendicular to the polarization axis $X_{O}$ ). Nevertheless, from the sole information contained in the coherency matrix, such situations are indistinguishable from a linearly polarized state with a well-defined direction of propagation.

In analogy to the information provided by the eigenvalues $\left(\lambda_{1}, \lambda_{2}, \lambda_{3}\right)$ of $\mathbf{R}$, which can alternatively be represented by the intensity $I$ together with two meaningful nondimensional parameters such as the indices of polarimetric purity $\left(P_{1}, P_{2}\right)$, it is worth exploring the possibility of finding an additional parameter $P_{l}$ that, together with $I$ and $P_{d}$, provides an alternative and meaningful view of the information given by the principal intensities $\left(a_{1}, a_{2}, a_{3}\right)$.

Let us now observe that an interesting parameter that is commonly used in 2D representations of polarized light is the so-called degree of linear polarization, defined as the ratio between the intensity of the linearly polarized portion and the total intensity of the state. By taking advantage of the analogies between the structures of $\left(\lambda_{1}, \lambda_{2}, \lambda_{3}\right)$ and $\left(a_{1}, a_{2}, a_{3}\right)$, we define the degree of linear polarization $P_{l}$ as the nondimensional parameter

$$
P_{l} \equiv \frac{a_{1}-a_{2}}{a_{1}+a_{2}+a_{3}},
$$

whose possible values are limited by $0 \leqslant P_{l} \leqslant P_{d}\left(P_{d} \leqslant 1\right)$. The minimum value $P_{l}=0 \quad\left(a_{1}=a_{2}\right)$ corresponds to a state with an intensity ellipsoid of revolution with semiaxes $\left(a_{1}, a_{1}, a_{3}\right)$, so that its intersection with the plane perpendicular to the average direction of propagation $\mathbf{k}$ is a circumference of radio $a_{1}$ (zero degree of linear polarization). Regardless to the value of $P_{d}, P_{l}=0$ corresponds to states with equal principal intensities in the plane perpendicular to $\mathbf{k}$. When, in particular, $P_{d}=0$, the intensity ellipsoid is a sphere and 
corresponds to a 3D unpolarized state. The maximum value $P_{l}=1$ entails $P_{d}=1$ and corresponds to a linearly polarized pure state. Furthermore, in the case of $2 \mathrm{D}$ states $\left(P_{d}=1\right), P_{l}=$ $\sqrt{s_{1}^{2}+s_{2}^{2}} / s_{0}$, as corresponds to the natural and commonly used definition of the degree of linear polarization. Thus the inspection of the values of $P_{l}$ for all the possible 3D states of polarization shows that $P_{l}$ gives an appropriate and consistent measure of the degree of linear polarization.

Consequently, leaving aside the intrinsic physical meaning of $\left(a_{1}, a_{2}, a_{3}\right)$ as the semiaxes of the intensity ellipsoid, this physical information can alternatively be represented by the set of quantities $\left(I, P_{l}, P_{d}\right)$, whose physical interpretation is particularly appropriate for the study and analysis of threedimensional states of polarization.

\section{CONCLUSIONS}

At a given point in the space, the second-order state of polarization of an arbitrary electromagnetic wave is characterized by means of the corresponding coherency matrix $\mathbf{R}$, which can be interpreted in terms of a set of nine well-defined parameters, namely [21], the principal intensities $\left(a_{1}, a_{2}, a_{3}\right)$; the angular momentum $\mathbf{n} \equiv\left(n_{1}, n_{2}, n_{3}\right)$, and the set of three orientation angles $(\varphi, \alpha, \beta)$. This parameterization constitutes an adequate framework for the analysis and interpretation of any three-dimensional state of polarization through the corresponding arbitrary and characteristic decompositions.

A degree of directionality $P_{d}$ has been defined which gives an appropriate and consistent measure of the stability of the direction of propagation of the state of polarization represented by $\mathbf{R}$. In addition, another nondimensional parameter $P_{l}$ representative of the degree of linear polarization has been defined, which together with the intensity $I$ and the degree of directionality $P_{d}$, constitutes a set of meaningful parameters that contains complete information about the intensity ellipsoid in an alternative way to that provided by the semiaxes $\left(a_{1}, a_{2}, a_{3}\right)$.

Together with the integer parameter $r \equiv \operatorname{rank} \mathbf{R}$, which determines the number of different pure components in the arbitrary decomposition, we have found that the integer parameter $t \equiv \operatorname{rank}[\operatorname{Re}(\mathbf{R})]$ plays a fundamental role in the physical interpretation of the three-dimensional states of polarization. $t=1$ corresponds to pure states with linear polarization $\left(P_{l}=P_{d}=1\right)$. As noted above, despite that the state can be considered to have a well-defined direction of propagation, such a direction of propagation is not determined by $\mathbf{R}$ (although it is true that the experimentalists usually have complementary information enough to determine it).

When $t=2$, the state $\mathbf{R}$ is reduced to a conventional two-dimensional state of polarization $\left(P_{l}<P_{d}=1\right)$, with a well-defined direction of propagation determined by $\mathbf{R}$. The arbitrary and the characteristic decompositions of $\mathbf{R}$ correspond to those of two-dimensional states and, in particular, the arbitrary decomposition is necessarily composed of two pure states with the same direction of propagation. Moreover, the characteristic decomposition has the well-known form of a superposition of a pure state and an unpolarized twodimensional state.

In the case that $t=3$, even if $r=2$, it is no longer possible to assign a well-defined direction of propagation to the state $\mathbf{R}\left(P_{l} \leqslant P_{d}<1\right)$, so that at least two pure arbitrary components have different propagation directions. Moreover, the characteristic decomposition adopts particular forms depending on the value of an additional auxiliary integer parameter.

The case study presented in Sec. III clarifies the interpretation and the role played by the set of two indices of polarimetric purity $\left(P_{1}, P_{2}\right)$ as physically invariant quantities that give nondimensional appropriate measures of the structure of purity of a state $\mathbf{R}$, beyond the overall information provided by the degree of polarimetric purity $P_{(3)}=\sqrt{3 P_{1}^{2}+P_{2}^{2}} / 2$.

The transformation of the coherency matrix performed through the appropriate rotation of the reference frame provides the intrinsic coherency matrix $\mathbf{R}_{O}$ characterized by the set of six parameters $\left(a_{1}, a_{2}, a_{3} ; n_{1}, n_{2}, n_{3}\right)$. The physical information contained in $\mathbf{R}_{O}$ can also be represented through the following alternative set of parameters $\left(I, P_{1}, P_{2}, P_{d}, P_{n}, n\right)$ with a clear and direct physical interpretation, namely, the intensity $I$; the degree of polarization $P_{1}$; the second index of polarimetric purity $P_{2}$ (i.e., the relative portion of the power of the wave obtained once the 3D unpolarized portion has been subtracted); the degree of directionality $P_{d}$; the degree of linear polarization $P_{l}$; and the magnitude $n$ of the angular momentum $\mathbf{n}$.

In summary, the approach presented, we think, constitutes a useful tool for the study, representation, and interpretation of the complete variety of three-dimensional states of polarization in terms of appropriate and well-defined physical parameters.

\section{ACKNOWLEDGMENTS}

This research was supported by Ministerio de Economía y Competitividad, Grant No. FIS2011-22496, and by Gobierno de Aragón, group E99.
[1] J. C. Samson, Geophys. J. R. Astron. Soc. 34, 403 (1973).

[2] R. Barakat, Opt. Commun. 23, 147 (1977).

[3] R. Barakat, Opt. Acta 30, 1171 (1983).

[4] T. Setälä, A. Shevchenko, M. Kaivola, and A. T. Friberg, Phys. Rev. E 66, 016615 (2002).

[5] T. Setälä, M. Kaivola, and A. T. Friberg, Phys. Rev. Lett. 88, 123902 (2002).
[6] J. J. Gil, J. M. Correas, P. A. Melero, and C. Ferreira, Monog. Sem. Mat. G. Galdeano 31, 161 (2004). Available from http://www.unizar.es/galdeano/actas_pau/PDFVIII/ pp161-167.pdf

[7] J. Ellis and A. Dogariu, Opt. Commun. 253, 257 (2005).

[8] A. Luis, Opt. Commun. 253, 10 (2005).

[9] A. Luis, Phys. Rev. A 71, 063815 (2005). 
[10] C. Brosseau and A. Dogariu, in Symmetry Properties and Polarization Descriptors for an Arbitrary Electromagnetic Wavefield, edited by E. Wolf, Progress in Optics Vol. 49 (Elsevier, New York, 2006), Chap. 4.

[11] F. T. Hioe, J. Mod. Opt. 53, 1715 (2006).

[12] M. R. Dennis, J. Opt. Soc. Am. A 24, 2065 (2007).

[13] J. J. Gil, Eur. Phys. J. Appl. Phys. 40, 1 (2007).

[14] T. Setälä, K. Lindfors, and A. T. Friberg, Opt. Lett. 34, 3394 (2009).

[15] J. C. Petruccelli, N. J. Moore, and M. A. Alonso, Opt. Commun. 283, 4457 (2010).

[16] I. San José and J. J. Gil, Opt. Commun. 284, 38 (2011).

[17] O. Gamel and D. F. V. James, Phys. Rev. A 86, 033830 (2012).

[18] J. M. Auñón and M. Nieto-Vesperinas, Opt. Lett. 38, 58 (2013).

[19] A. V. Novitsky, arXiv:1308.2848v1 (2013).

[20] O. Gamel and D. F. V. James, J. Opt. Soc. Am. A 31, 1620 (2014).

[21] M. R. Dennis, J. Opt. A: Pure Appl. Opt. 6, S26 (2004).

[22] J. H. Hannay, J. Mod Opt. 45, 1001 (1998).

[23] T. Saastamoinnen and J. Tervo, J. Mod. Opt. 51, 2039 (2004).

[24] T. Carozzi, R. Karlsson, and J. Bergman, Phys. Rev. E 61, 2024 (2000).

[25] J. J. Gil and I. San José, Opt. Commun. 283, 4430 (2010).

[26] C. J. R. Sheppard, J. Opt. Soc. Am. A 28, 2655 (2011).

[27] C. J. R. Sheppard, Opt. Lett. 37, 2772 (2012).

[28] J. Ellis, A. Dogariu, S. Ponomarenko, and E. Wolf, Opt. Lett. 29, 1536 (2004).

[29] R. M. A. Azzam, J. Opt. Soc. Am. A 28, 2279 (2011).
[30] C. Ding, Y. Cai, Y. Zhang, and L. Pan, J. Opt. Soc. Am. A 29, 1078 (2012).

[31] U. Fano, Rev. Mod. Phys. 29, 74 (1957).

[32] J. J. Gil and I. San José, J. Opt. Soc. Am. A 30, 1078 (2013).

[33] R. Dändliker, P. Tortora, L. Vaccaro, and A. Nesci, J. Opt. A: Pure Appl. Opt. 6, S18 (2004).

[34] J. Ellis and A. Dogariu, Phys. Rev. Lett. 95, 203905 (2005).

[35] J. Ellis, A. Dogariu, S. Ponomarenko, and E. Wolf, Opt. Commun. 248, 333 (2005).

[36] P. Réfrégier, M. Roche, and F. Goudail, J. Opt. Soc. Am. A 23, 124 (2006).

[37] C. Macías-Romero, M. R. Foreman, and P. Török, Opt. Express 19, 25066 (2011).

[38] P. Réfrégier, V. Wasik, K. Vynck, and R. Carminati, Opt. Lett. 39, 2362 (2014).

[39] A. Luis, Phys. Rev. A 71, 023810 (2005).

[40] N. I. Petrov, Laser Phys. 18, 522 (2008).

[41] T. Voipio, T. Setälä, A. Shevchenko, and A. T. Friberg, Phys. Rev. A 82, 063807 (2010).

[42] A. Shevchenko, T. Setälä, and A. T. Friberg, Appl. Opt. 51, C44 (2012).

[43] J. J. Gil, I. San José, and R. Ossikovski, J. Opt. Soc. Am. A 30, 32 (2013).

[44] J. J. Gil, J. Appl. Remote Sens. 8, 081599 (2014).

[45] F. Snik, J. Craven-Jones, M. Escuti, S. Fineschi, D. Harrington, A. De Martino, D. Mawet, J. Riedi, and J. S. Tyo, Proc. SPIE 9099, 90990B (2014). 